\title{
Comparison of two finite element homogenization prediction approaches for through thickness thermal conductivity of particle embedded textile composites
}

\author{
Hang Yu ${ }^{\mathrm{a}, \mathrm{b}}$, Dirk Heider ${ }^{\mathrm{a}, \mathrm{c}}$ and Suresh Advani ${ }^{\mathrm{a}, \mathrm{b}, *}$ \\ ${ }^{a}$ Center for Composite Materials, University of Delaware, Newark \\ ${ }^{\mathrm{b}}$ Department of Mechanical Engineering, University of Delaware, Newark \\ ${ }^{\mathrm{c}}$ Department of Electrical and Computer Engineering, University of Delaware, Newark
}

\begin{abstract}
A detailed three dimensional finite element model was developed to predict the through-thickness thermal conductivity of a textile composite structure consisting of a woven fabric and matrix in our previous work [1] in which conductive particles are randomly distributed in the matrix in the unit cell already containing the woven fabric and the effective thermal conductivity of this three-phase system is numerically evaluated. In this paper, results of this three-component system are compared with a two-component system in which the thermal conductivity of the matrix and particles is homogenized by a finite element approximation under the same thermal conditions. A parametric study of this comparison is conducted over different volume fractions and thermal conductivities of both textile fabric and loaded particles and its efficacy is discussed. Typically such composites could be coated with a conductive skin on the surface. Thus, the role of the skin and fiber conductivities in enhancing heat transfer is compared for this structure. The proposed approach of finite element homogenization of particle-matrix medium can be used to conduct a preliminary assessment of through-thickness thermal conductivity of such three-component composite systems with fabrics, particles and matrices with and without the presence of a skin layer.
\end{abstract}

Keywords: woven fabrics, finite element method, thermal conductivity, three component systems, particle suspensions

\section{Introduction}

Textile composites continue to gain market share in fabrication of composite structures since the last decade [2-5]. Two dimensional woven fabrics [6, 7] are commonly used in composite structures because of their light weight, low fabrication costs, ease of handling, high adaptability and tailorability. Different types of fibers, including carbon, glass, aramid and various polymer matrix materials have been used to create these heterogeneous composite laminates for different applications. Also, different woven architectures (e.g. weaving [8], braiding [9], knitting [10]) for textile fabrics can be employed to tailor the composite physical and mechanical properties. Over the last few decades, a number of predictive models have been proposed to assess effective properties of composites based on constituent materials and their properties.

Predictive models to find effective properties of a composite material range from very

* Corresponding author. Email address: advani@udel.edu. 
simple weighted average of the constituent material properties such as the rule of mixtures to very complicated formulations that account for spatial and orientation variation of the fibers in the system [11-14]. The effective properties have also been determined using numerical methods which can address the specifics of the geometry [15-19] and can be integrated with macroscopic analysis [20].

Effective properties determined from an analytic constitutive relationship can be readily used in stress, thermal or electrical analysis of the composite but no models that can accurately predict the effective thermal properties for particle embedded woven structures exist to the authors' knowledge. In this work, we will focus on through-thickness thermal conductivity prediction of woven fabric with particle loaded polymer matrix [21]. Currently there is no closed form equation that can accurately describe the effective through thickness thermal conductivity of the composite, given the thermal conductivity of the fibers, matrix and particles over a range of fiber and particle volume fractions. Our approach is to predict the effective thermal conductivity using very detailed finite element models of a unit cell that represent the fiber configuration and geometry, covering a range of particle and fiber thermal conductivities and volume fractions. The results are compared with a simplified two-phase model (fiber and matrix) in which the mixture of particles and matrix are homogenized as one phase by using finite element approximation to find the homogenized matrix value to be used in the simplified two-phase model. Models with different volume fractions of fibers and particles over a range of thermal properties are compared in terms of the efficacy of the finite element homogenization approach. The role of a conductive skin as a coating on the surface is evaluated for the effective through-thickness thermal conductivity of the three-phase model. The results of the parametric study can be used to provide guidelines for material selection and design.

\section{3D geometric model of woven fabric}

The effective through-thickness thermal conductivity of woven fabric is highly dependent on the constituent properties and the reinforcement architecture. The fabric geometry in microscopic detail will influence the effective properties of the composite. It is important to mesh the 3D geometric model to describe the fiber continuity and undulation without simplifying the architecture. Here Sheng and Hoa's approach [22] was adopted and weft and warp yarns were assumed to have elliptical cross sections as shown in Fig. 1.

The central path of the fiber crimp is taken to be a repetitive combination of elliptical segment $K J$ and rectilinear segment $J M$ to simplify fiber spatial orientation. The undulated segment $K J$ of the yarn is described by an elliptical equation

$$
\frac{(x-m)^{2}}{w^{2}}+\frac{(y-n)^{2}}{t^{2}}=\frac{1}{4}, \quad\left(J_{l x} \leq x \leq J_{r x}\right)
$$

where $m, n$ represent the central coordinates of the cross section of the yarn on the $\mathrm{XY}$ plane; $J_{l x}, J_{r x}$ are the $x$ coordinates of the two tangent points of woven yarns; $w, t$ represent the major axis (width) and minor axis (thickness) of the fabric cross section, respectively. The rectilinear segment JM of the yarn between two elliptical segments is given by a linear equation

$$
y=k x+d,\left(J_{r x} \leq x \leq M_{x}\right)
$$


where $k$ is the slope of the rectilinear segment $J M, d$ is a constant and $M$ is the center point of the linear path.

The coordinates of the tangent point $J$ connecting elliptical segment and linear segment can be written as

$$
J\left(m+E, n-t \sqrt{\frac{1}{4}-\frac{E^{2}}{w^{2}}}\right)
$$

Thus the slope of the segment JM is determined by the two points $J, M$ as

$$
k_{J M}=\frac{M_{y}-J_{y}}{M_{x}-J_{x}}
$$

Also from the elliptical eqn. (1), the slope of the tangent line at the point $J$ can be expressed as:

$$
k_{J M}=\left.\frac{d y}{d x}\right|_{x=m+T}=\frac{E m / w^{2}}{\sqrt{\frac{1}{4}-\frac{E^{2}}{w^{2}}}}
$$

By combining Eqns. (4) and (5), the distance $E$ and the slope of the segment $J M$ can be calculated. An arbitrary distance $t_{d}=w / 10$ is designated as the distance from the yarn to the bottom and top surface of the model.

The three dimensional geometry of the reinforcing fabric for a woven fabric composite can be fully described by the previously defined geometric parameters and the spatial orientation of the yarn. In this paper, the fiber volume fraction is varied by changing the cross sectional area of the yarns and the thickness $b$ of the cell while maintaining the ratio $\mathrm{w} / \mathrm{t}=2$, and the unit cell width and length equal to $a$. With the above general approach, 3D finite element model of the geometric arrangement shown in Fig. 2 can be generated for further analysis. This approach also makes it easier to study other geometric parameters of the yarns and their influence on the effective composite property.

A set of 3D fabric models were developed and meshed using ANSYS software as the tool of Finite Element Analysis with formulated macros scripted using Mechanical APDL. The element type used for the geometry mesh is SOLID70 which is a 3-D thermal solid element which has eight nodes with a single degree of freedom-- the temperature at each node. The curved shape for the yarn undulation was approximated by piecewise line segments for the ease of line generation, hence there is a small gap of width equal to w/40 to avoid yarn geometric intervene at the nearest region.

The ANSYS finite element software solves the steady state heat diffusion equation to evaluate the temperature field in the whole domain. Each element is assigned a thermal conductivity value of the fiber, matrix or particle. The boundary conditions prescribed are insulated along the four sides of the unit cell and constant temperature along the top and the bottom face ( $T_{1}$ and $T_{2}$ respectively). Once the temperature field is evaluated, the heat flow $q$, which should be the same over the top and bottom face is calculated by integrating the heat flux across each element on the face. Then the effective through-thickness thermal conductivity can be calculated using Fourier's law as follows,

$$
q=-k_{e f f} A\left(T_{1}-T_{2}\right) / b,
$$

In which $q$ is the calculated heat transfer rate over the entire surface area (A) across the unit cell thickness $b ; T_{1}$ and $T_{2}$ are the temperature imposed as boundary conditions on the top and the bottom surface of the unit cell. Eqn. (6) allows one to calculate the effective 
through-thickness thermal conductivity $k_{e f f}$.

\section{Two-phase finite element model of particle and matrix}

The procedure in this study is to calculate the effective thermal property of the mixture of matrix containing particles which are randomly placed, then use this effective thermal conductivity as one homogenized phase where the other phase in the composite is the fiber conductivity. An example of the mesh of a unit cell containing particles and matrix is shown in Fig. 3. Particle sizes are determined based on the element size control in the mesh generation, and the average particle volume is around $0.00002 \sim 0.0002 \mathrm{~mm}^{3}$, which is close to the range of particle size in some applications, like glass bead and copper particles [23, 24].

However as the particles are assigned randomly in the unit cell, the influence of random placement is evaluated by calculating the assemblage average from five different runs with the same particle volume fraction. The approach is repeated for different particle volume fraction from $10 \%$ to $50 \%$. The results are shown in Table I. Fig. 4 shows the comparison between the finite element prediction and the rule of mixture results.

Table I. Effective through-thickness thermal conductivity of two-phase finite element models with particles and matrix for different particle volume fractions and thermal conductivities

\begin{tabular}{c|c|c|c}
\hline \multirow{2}{*}{$k_{e f f} / k_{m}$} & $k_{p} / k_{m}=10$ & $k_{p} / k_{m}=100$ & $k_{p} / k_{m}=1000$ \\
\cline { 2 - 4 } Particle $\mathrm{V}_{\mathrm{fp}}$ & \multicolumn{3}{|c}{ Mean \pm Standard deviation } \\
\hline $10 \%$ & $1.54 \pm 0.001$ & $4.08 \pm 0.01$ & $23.40 \pm 0.12$ \\
$20 \%$ & $2.23 \pm 0.002$ & $10.77 \pm 0.03$ & $91.66 \pm 0.39$ \\
$30 \%$ & $3.02 \pm 0.003$ & $19.59 \pm 0.04$ & $182.16 \pm 0.49$ \\
$40 \%$ & $3.91 \pm 0.006$ & $29.83 \pm 0.07$ & $286.96 \pm 0.77$ \\
$50 \%$ & $4.85 \pm 0.01$ & $40.95 \pm 0.10$ & $400.63 \pm 1.06$ \\
\hline
\end{tabular}

The result shows that the effective thermal conductivity of particle-matrix system as a function of particle volume fraction $v_{f p}$. The standard deviations shown above indicate that the standard deviation is negligibly small for the different particle location choices for the same particle volume fraction. It also indicates that a large through-thickness thermal conductivity improvement can be achieved by adding a large amount of conductive particles in the matrix, however the rule of mixtures always seems to over predict the result at these volume fractions of particles. The rule of mixture approximation becomes reasonable at high volume fractions and for lower thermal conductivity ratios but is grossly incorrect at lower particle volume fractions which is what is used in three phase fibrous composite systems as $50 \%$ of the volume is occupied by the fibers, and the remaining is shared between resin and matrix. The studies conducted limit the fiber volume of particles to $30 \%$ and hence the value predicted by the FE models will be used when needed. 


\section{Woven fabric system with effective particle-matrix phase}

A set of two-phase woven fabric composite models was developed with ANSYS software for numerical simulations. The two phases are the fibers and the effective matrix. Based on the model generation scheme in section 2, we vary the fabric cross section area while maintaining the ratio $w / t=2$ and $b / w=1.2$. The total number of elements in the finite element model is between 199,993 and 1,028,755 depending upon the fabric volume fractions and cross sections. The number of elements was decided to guarantee a converged solution. The fabric volume fraction ranges from $12 \%$ to $68 \%$ considering the fabric geometric constraint in the unit cell with a finite length and width. Three models with different fiber volume fractions are shown in Fig. 5.

With the fixed yarn distance $\mathrm{L}+\mathrm{E}$, as shown in Fig. 1, the highest fabric volume fraction is reached for the case (3) in Fig. 5. Length and width have the same value $a$. It is assumed that all yarns and matrix have isotropic and homogeneous thermal conductivities in the current study. 


\section{Three-phase numerical model with woven fabric and conductive particles within the matrix}

A number of studies have been reported on the prediction of mechanical or thermal property characterization with only particles embedded in the matrix [25-27] or with only fiber reinforced composite materials, but there are few that have studied three component systems - particle, fiber and matrix together [28-30]. In our work we investigate a composite system which contains plane woven fabric with randomly distributed conductive particles in the matrix to quantify their respective influence on the through-thickness thermal conductivity of the composite. The intent is to predict the through-thickness thermal conductivity of the three-phase composite structure and compare the results with two phase numerical models in which the matrix conductivity is represented by the homogenized particle and matrix conductivity from the FE calculation results shown in Table I. The goal is to quantify the error introduced in the predictions if the conductivity of the particles in the matrix is homogenized and represented as effective matrix conductivity in a two phase finite element model of fibers and matrix. The error is calculated by comparing the two-phase model results with the three-phase model in which the particles are separately represented with their own conductivity value.

Three-phase models are created by specifying the particle thermal conductivity value for a number of elements randomly dispersed within the matrix in the finite element model developed in the previous section. Particles are assumed to have isotropic thermal conductivity. The architectural complexities of woven fabric model make it difficult to quantify their constitutive properties by analytical approaches and numerical models are usually applied. As particles are represented by elements it was deemed important to conduct mesh refinement studies to ensure convergence of the effective thermal conductivity.

\subsection{Mesh refinement study}

The mesh refinement study is conducted to ensure that the numerical models reach an acceptable level in terms of convergence and accuracy for engineering solutions. So the key is to provide sufficient accuracy without the penalty of high computational costs. Fig. 6 shows one of the models where the three-phase structure has particle volume fraction of $10 \%$ and woven fabric has a volume fraction of $48 \%$.

The mesh refinement operation can divide one particle element into multiples ones, therefore those nodal values of the particle elements can provide higher accuracy when the particle element properties are significantly different from that of the matrix elements.

Based on the mesh refinement study in [1], we decided to use the $1^{\text {st }}$ order mesh to perform the finite element analysis to predict the effective through-thickness thermal conductivity of the three-phase composite model.

\subsection{Three-phase numerical model results}

The mathematical formulation of the heat conduction problem is stated by Fourier's Law and the effective through-thickness thermal conductivity $k_{e f f}$ can be calculated for its steady-state heat transfer conditions with no internal heat source. The $k_{\text {eff }}$ reflects the heat conduction ability of the composite system across the thickness. We assume the heat flow 
within the plane is symmetric. Thus, for many repetitive cells at the interface between two cells the heat flow is balanced in the plane due to the repetitive feature in the plane which is represented by the insulated boundary condition. A constant temperature gradient is applied across the woven cell thickness.

The parametric numerical study is conducted for a range of particle volume fractions and thermal conductivities as well as for a range of woven fabric volume fractions and thermal conductivities. In the three-phase model, parametric studies are conducted with different particle thermal conductivities $k_{p}\left(k_{p} / k_{m}=10,100,1000\right)$, fabric thermal conductivities $k_{f}\left(k_{f} / k_{m}=2^{i}, i=0,1, \ldots, 11\right)$ and particle volume fractions $v_{f p}\left(v_{f p}=10 \% \sim 30 \%\right)$, and fabric volume fractions $v_{f} \quad\left(v_{f}=10 \% \sim 60 \%\right)$.

Fig. 7 shows the through-thickness $k_{\text {eff }}$ results with $38.79 \%$ fiber volume fraction for different $k_{f} / k_{m}$ ratios and $k_{p} / k_{m}$ ratios where $k_{f}, k_{p}$ and $k_{m}$ are the fiber, particle and matrix conductivity respectively. Usually the matrix conductivity is of the order of $0.1 \mathrm{~W} / \mathrm{mK}$ and fiber conductivity can range from the same order (glass fibers) to two to three orders higher (carbon fibers). Here the effective properties for the particle-matrix medium calculated in section 3 and shown in Fig. 4 are used for the results shown in Fig. 7.

As is shown in Fig. 7, the two-phase plane woven fabric model through-thickness thermal conductivity $k_{\text {eff } 2}$ increases nonlinearly as the fiber thermal conductivity increases, and the loading of the conductive particles can enhance the effective thermal conductivity for the composite system.

To understand if the homogenization solution of finite element method for the particle-matrix medium is a good approximation for the three-phase system which contains fibers, matrix and particles, a series of three-phase numerical models were developed and the effective thermal conductivity is predicted using a finite element simulation. These models contain elements that represent the fibers, matrix and particles and the effective thermal conductivity was calculated for this three phase system for various volume fractions and conductivities of fabric, matrix and particles and compared with fabric-effective matrix two-phase models to examine their accuracy. Fig. 8 shows the three-phase simulation results for the models that contain $38.79 \%$ volume fraction fiber and different volume fractions of distributed particles.

The results indicate that the effective through-thickness thermal conductivity of the three-phase model has a similar relation with fiber thermal conductivity compared to the models where the particles and matrix are homogenized as one phase, but the prediction results $k_{e f f 3}$ is smaller than $k_{e f f 2}$ which was obtained using homogenized particle and matrix medium. The combination of distributed conductive particles and the continuous fabric can form continuous conductive paths across the thickness to improve the through-thickness thermal conductivity. In three-phase models, the conductive particles are distributed randomly in the matrix phase and are separated by the continuous low conductivity matrix phase hence there is more resistance to heat flow across the thickness. However in the two-phase model in which the particles and matrix properties are homogenized as one phase, the isolated heat resistance of the matrix cannot be captured using the homogenization, hence the two-phase model overpredicts the heat transfer across the thickness. In the next section we quantify the overestimation from the homogenized two-phase model. 


\subsection{Model comparison}

We compared the finite element simulation of these three-phase models to the two-phase models containing fabric and a homogenized matrix using finite element approximation for the particle-matrix medium mentioned in section 4 in order to explore the efficacy of homogenization accuracy. For the case with $38.79 \%$ volume fraction of fibers, the comparison is shown in Fig. 9.

The comparison shows that for the case where the fiber volume fraction $v_{f}$ is low, the two-phase model including the homogenized matrix and particle phase always predicts a higher thermal conductivity than the three-phase models with fibers, matrices and particles, i.e. $k_{e f f 2} / k_{e f f 3}>1$. And the overestimation ratio is dependent on the thermal conductivity of particles and fibers.

However, if the fiber volume fraction is higher, the two-phase estimation does not necessarily overestimate the effective through-thickness thermal conductivity of the three-phase structure, as shown in Fig. 10.

For these cases, the two-phase model does not necessarily overestimate the effective thermal conductivity $\left(k_{e f f 2} / k_{e f f 3}>1\right)$ except for the case in which particle thermal conductivity is low, $k_{p} / k_{m}=10$. The underestimation for the cases where fiber volume fraction is high is due to the limited amount of matrix in the structure. As the volume fraction of the matrix is small, it does not construct the same barrier to weaken the heat transfer from particles to particles or fibers, so the finite element approximation of the particle and matrix system underestimates the particle-particle conductive interaction for these cases. Also, compared to the pure particle and matrix system modeled in section 3 where the particles are distributed anywhere within the matrix, the conductive particles can only be distributed in the empty regions where there are no fibers thus the particles have more chance of being in contact with increasing fiber volume fraction. This comparison can be used as an estimation of how the finite element approximation of particle volume fraction and conductivity can influence the prediction of through thickness thermal conductivity in the composite systems with textile fabrics.

\section{Three-phase model with surface coatings}

As the conductive pathways across the thickness are crucial for the heat to transfer between top and bottom surfaces, a conductive coating is applied on the model surfaces (which we will refer to as a skin layer) to show its influence on the effective through-thickness thermal conductivity. The conductive skin layer opens up additional pathways making the heat conduction more efficient than the case where there is no skin layer on the top and bottom surfaces. Fig. 11 shows the three-phase model with 35\% fiber volume fraction, $10 \%$ particle volume fraction and $10 \%$ skin on the surfaces.

Using the three phase model, three different skin thermal conductivities $k_{l}$ are compared and the effective through-thickness thermal conductivity is shown in Fig. 12.

Conductive skin has a dramatic influence on the composite conductivity. Also it is worth noting that for the case where there is "no fiber", particles are distributed everywhere within the matrix, and for the case with woven fabric reinforced, particles are squeezed into the space unoccupied by the fabric. So when a high volume fraction of conductive particles are 
embedded, the reinforcement of low-conductivity fiber could actually weaken the heat conduction by acting as high-resistance paths. With higher thermal conductivity of fibers, conductive particles are not only connected by themselves, but also by the continuous conductive fibers interlaced in the matrix to improve the conduction across the thickness. The multiple conductive ways provided by the combination of conductive skin, particles and interacted fabric can bring extra thermal efficiency for the heat conduction across the thickness. The nonlinear influence of the skin layer on enhancing the thermal conductivity as the particle thermal conductivity increases is shown in Fig. 13.

For example, when the skin layer is 100 times more conductive than the matrix, the effective thermal conductivity of the composite increases slightly less than an order of magnitude when the particle thermal conductivity is increased by an order of magnitude.

\section{Conclusions}

In this paper, a general geometric model to discretize a woven fabric within a unit cell is developed and its thermal modeling with three-phase woven composites is presented. The 3D model takes into consideration a comprehensive understanding of fabric geometric characteristics and distributed particles into the thermal analysis. The proposed thermal modeling uses a finite element approach to predict the through-thickness thermal conductivity of a unit cell with randomly distributed particles and matrix. The predicted results are used as a homogenization of the particle and matrix phases in the woven fabric model which contains fabric and a homogenized matrix particle phase. The comparison between the two-phase model and three-phase models show that the finite element model in which a homogenized particle-matrix phase is used overestimates the effective conductivity at relatively low fiber volume fraction and underestimates it for the case with very high fiber volume fraction. With conductive skin and fibers integrated in the three-phase structure, the effective through-thickness thermal conductivity can be improved by the high-efficiency of the thermal network.

\section{Acknowledgements}

This work is supported by the National Science Foundation Grant CMMI-0970002. 


\section{Appendices}

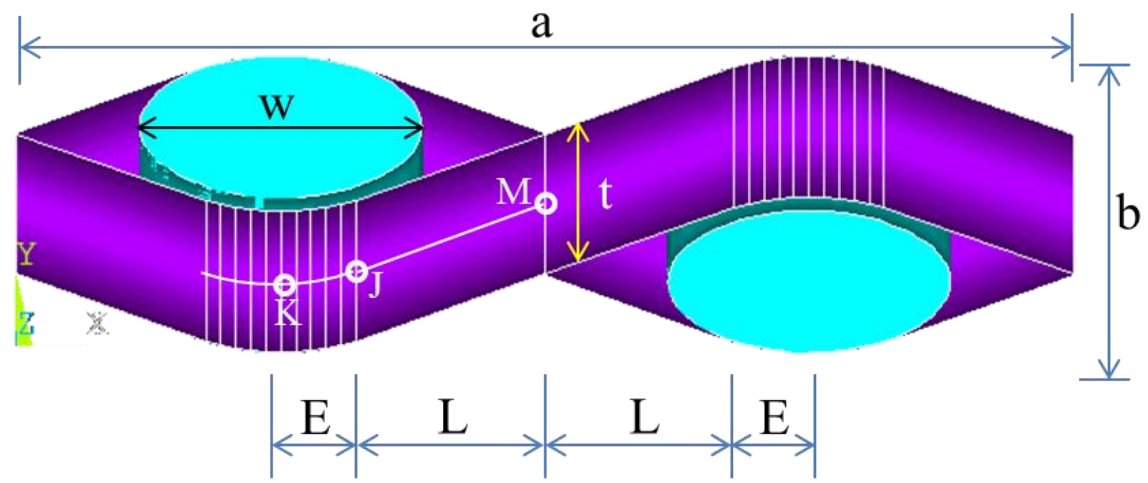

Fig. 1.

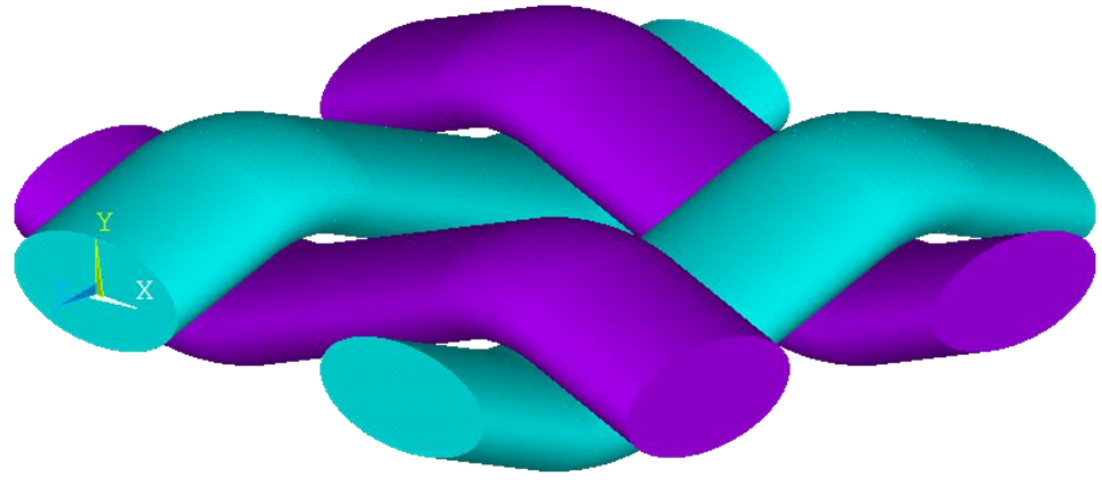

Fig. 2.

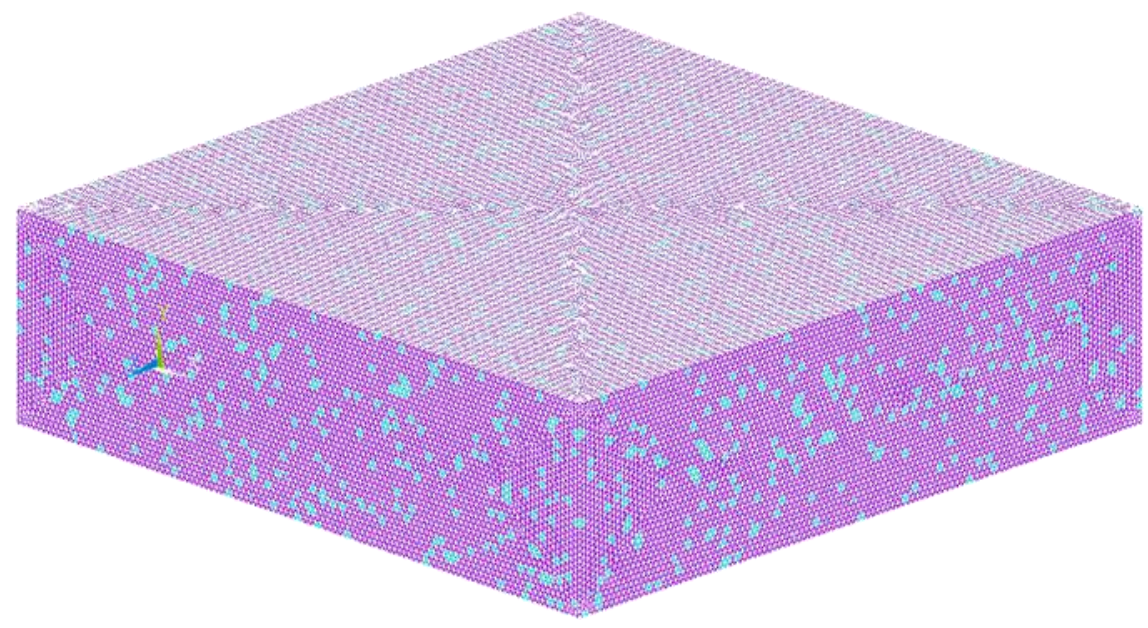

Fig. 3. 


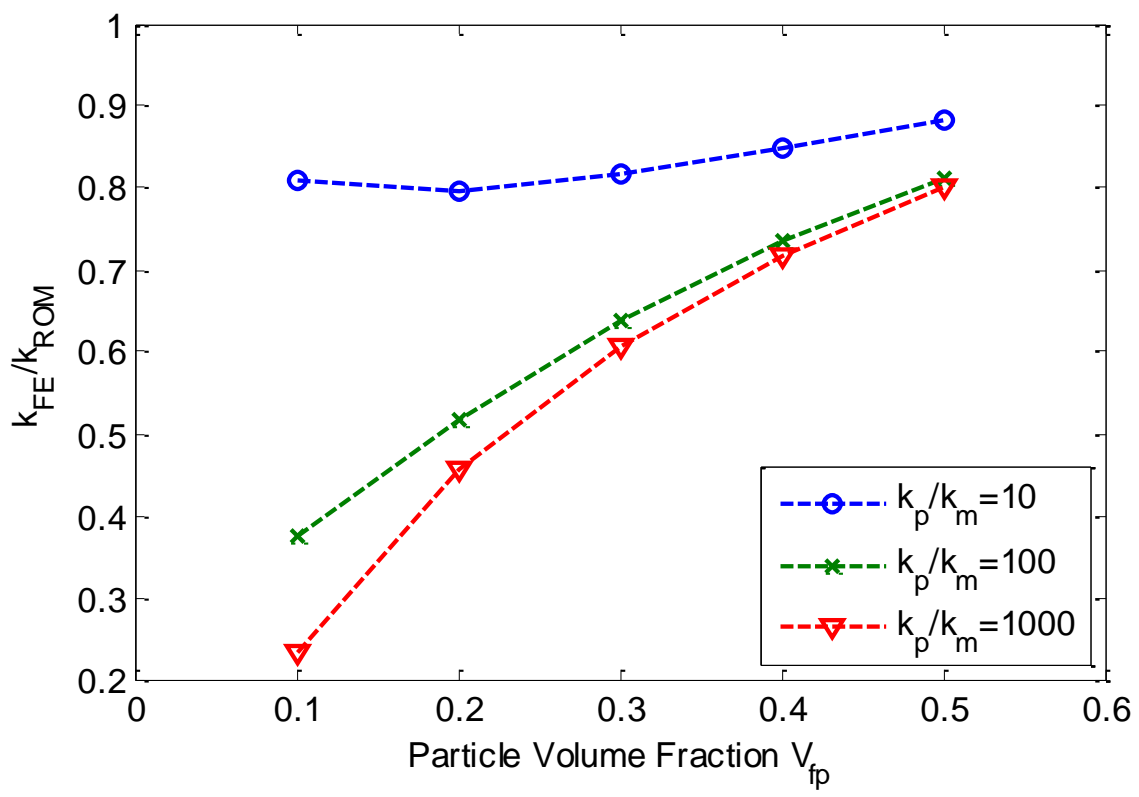

Fig. 4.

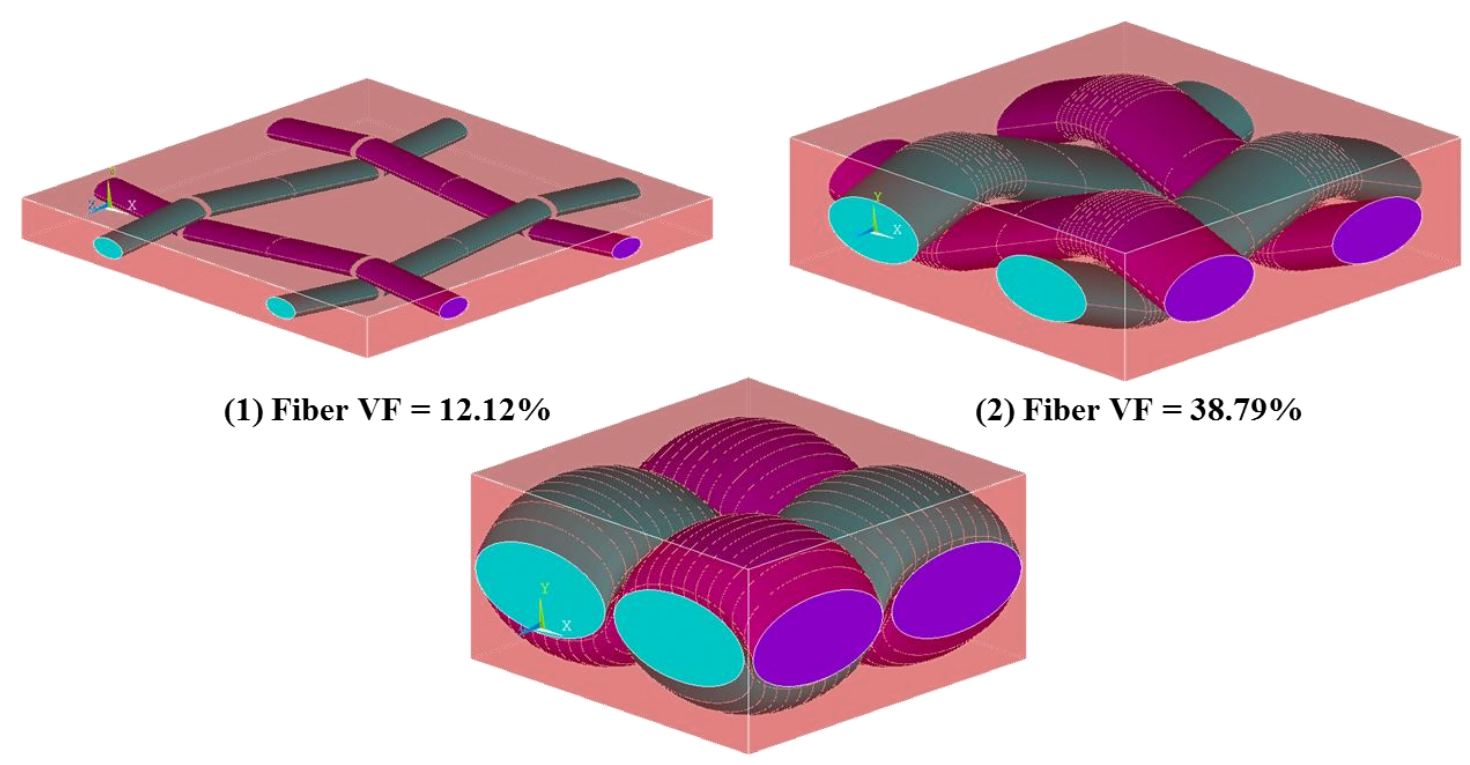

(3) Fiber $V F=67.87 \%$

Fig. 5. 


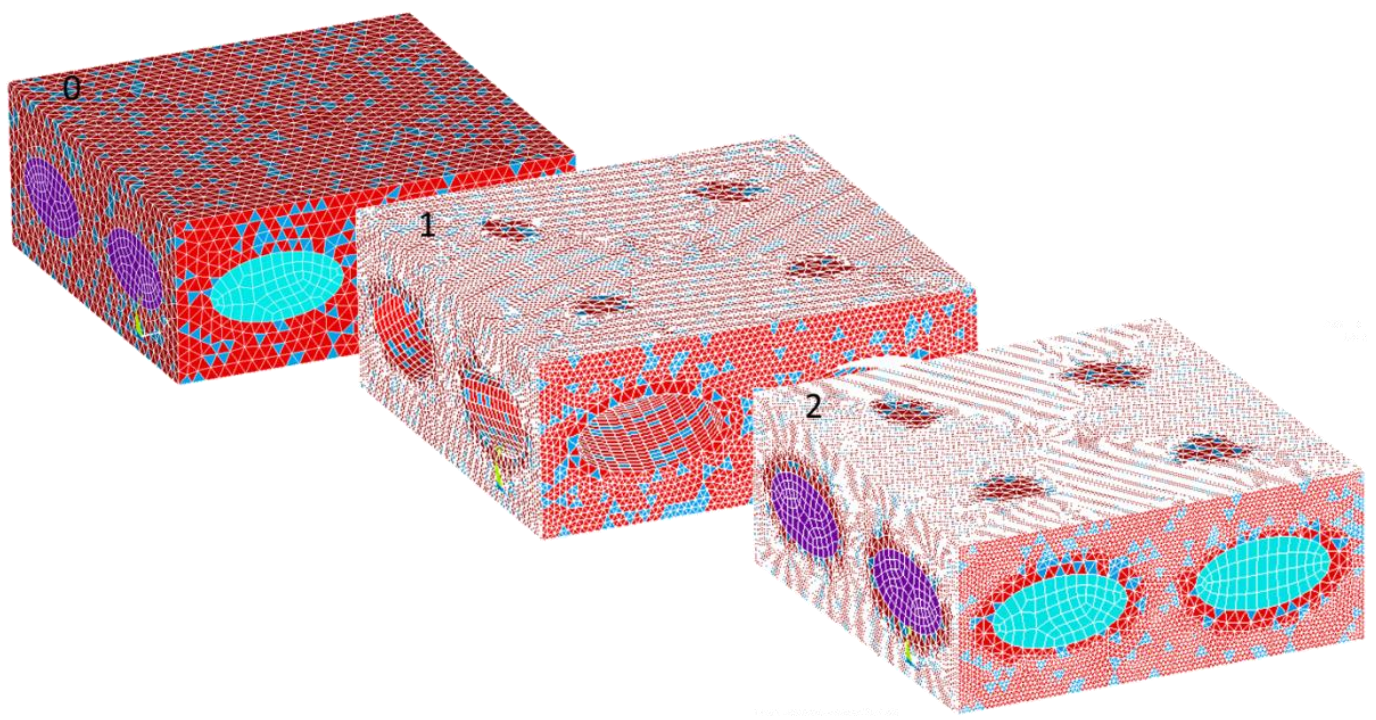

Fig. 6.

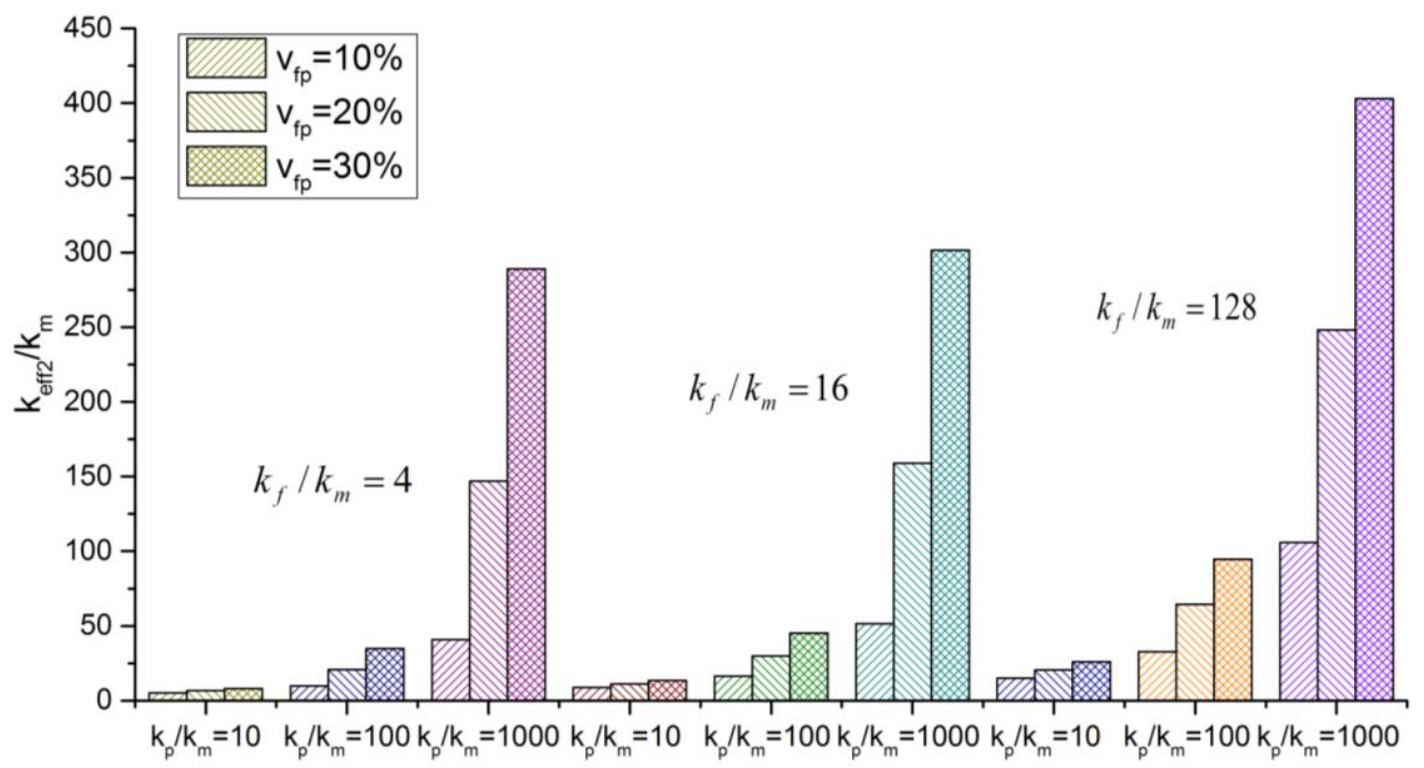

Fig. 7. 


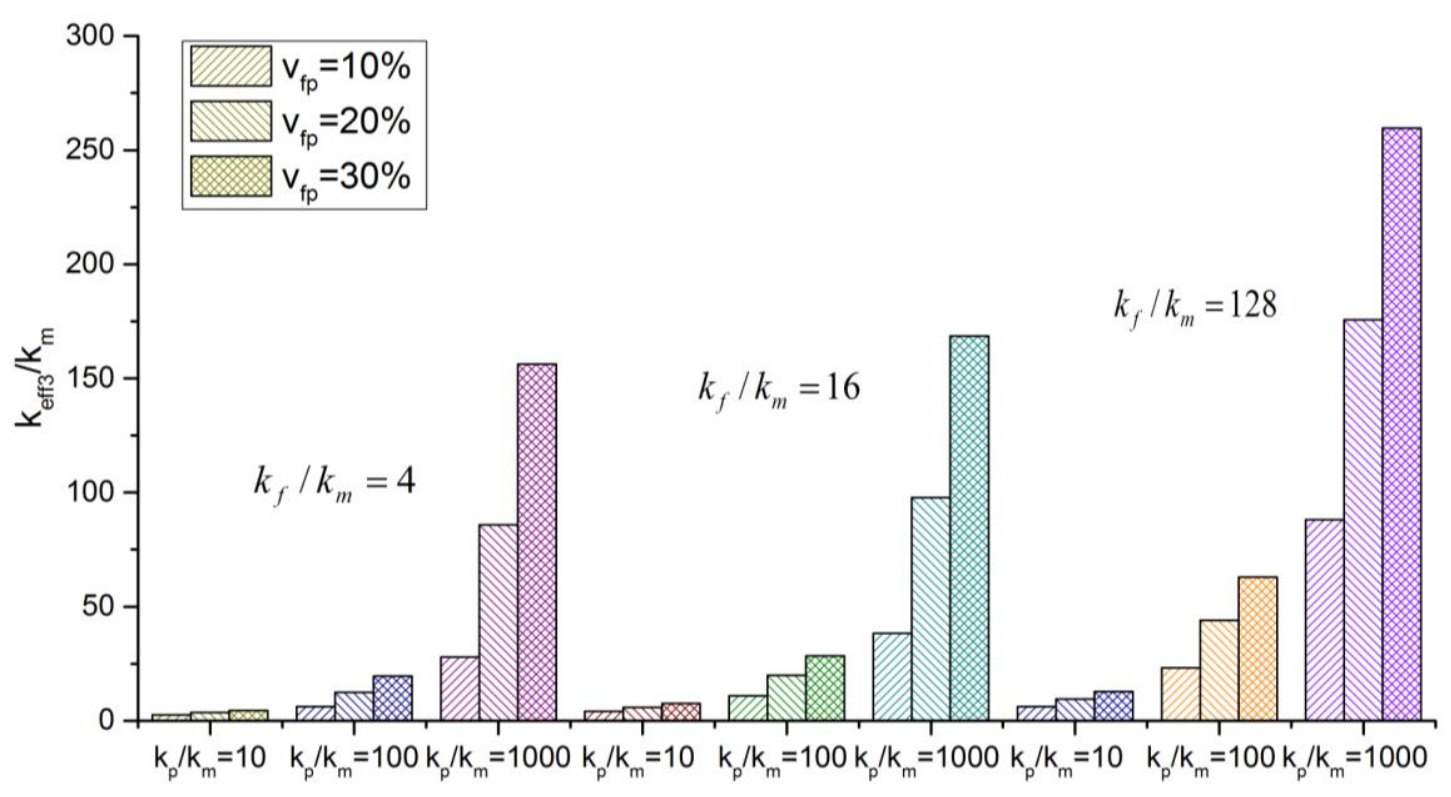

Fig. 8 .

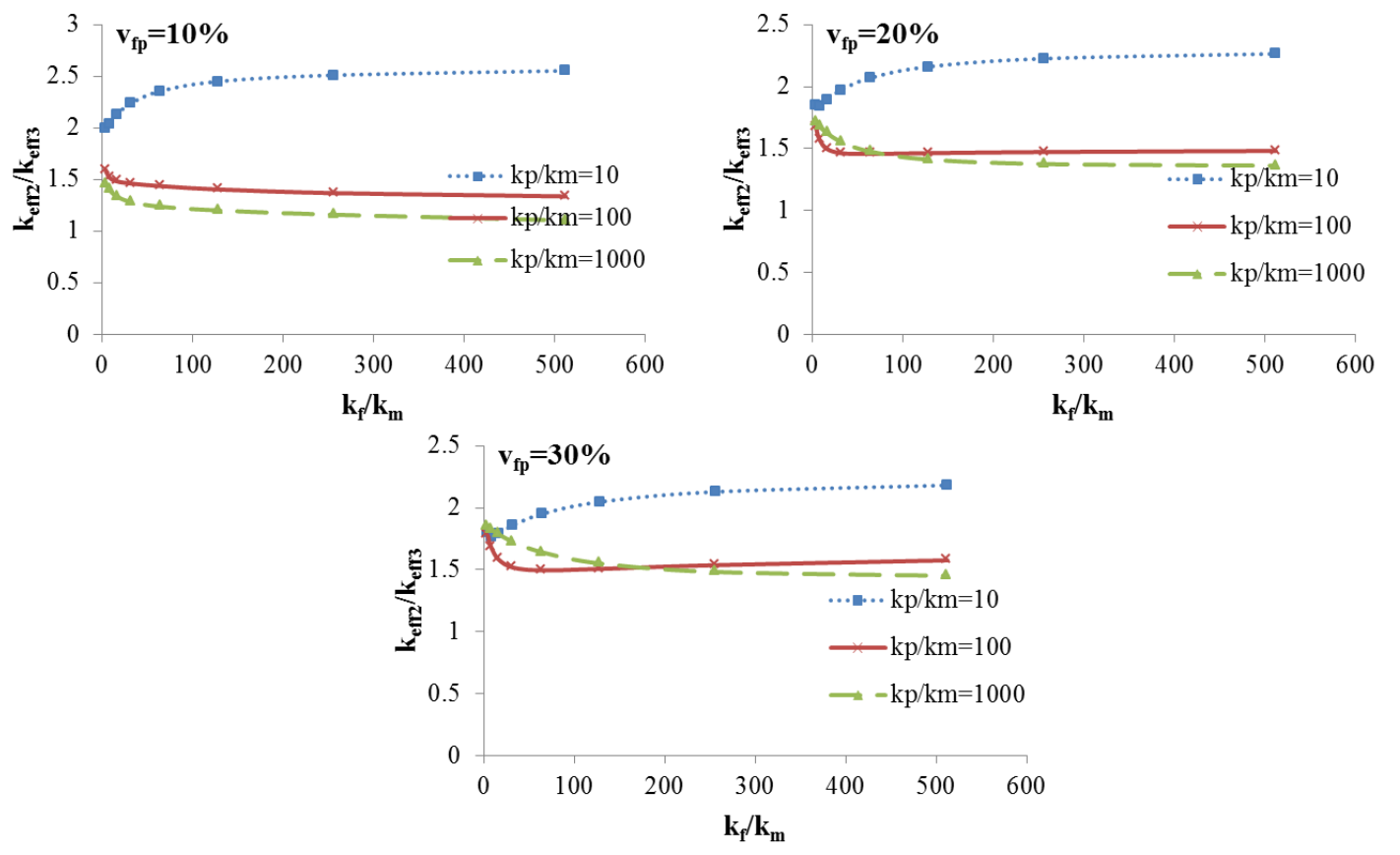

Fig. 9. 


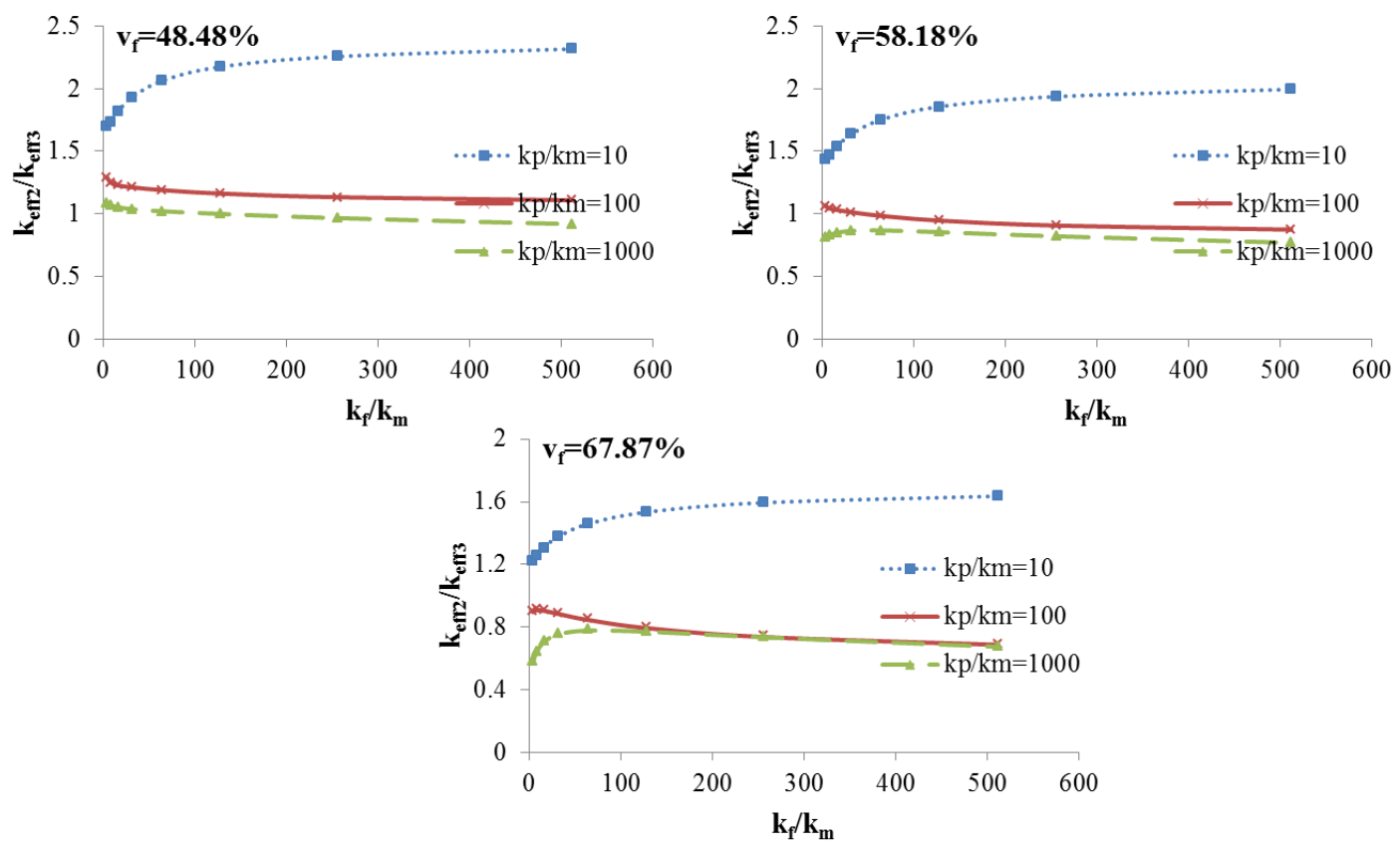

Fig. 10.

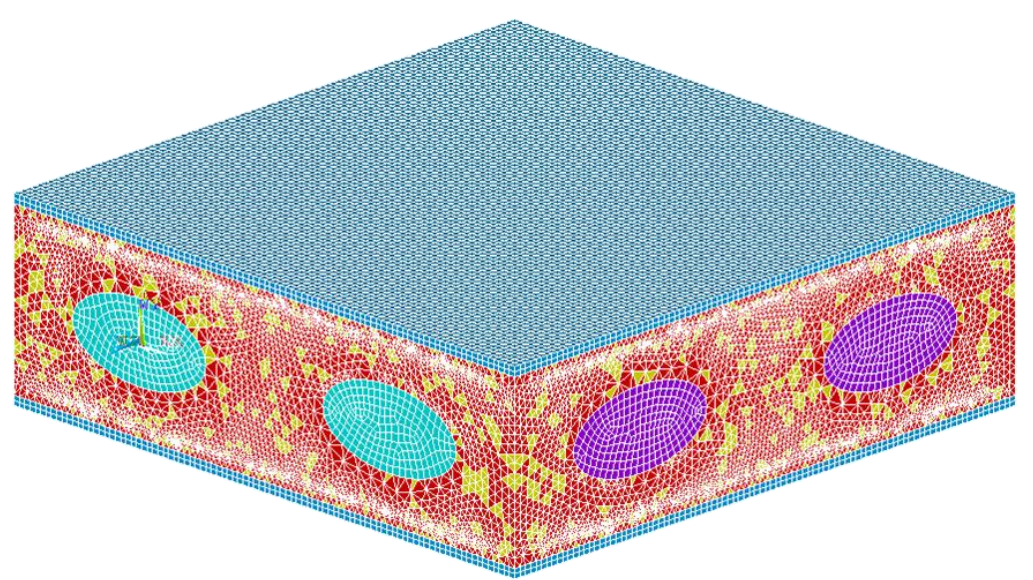

Fig. 11. 

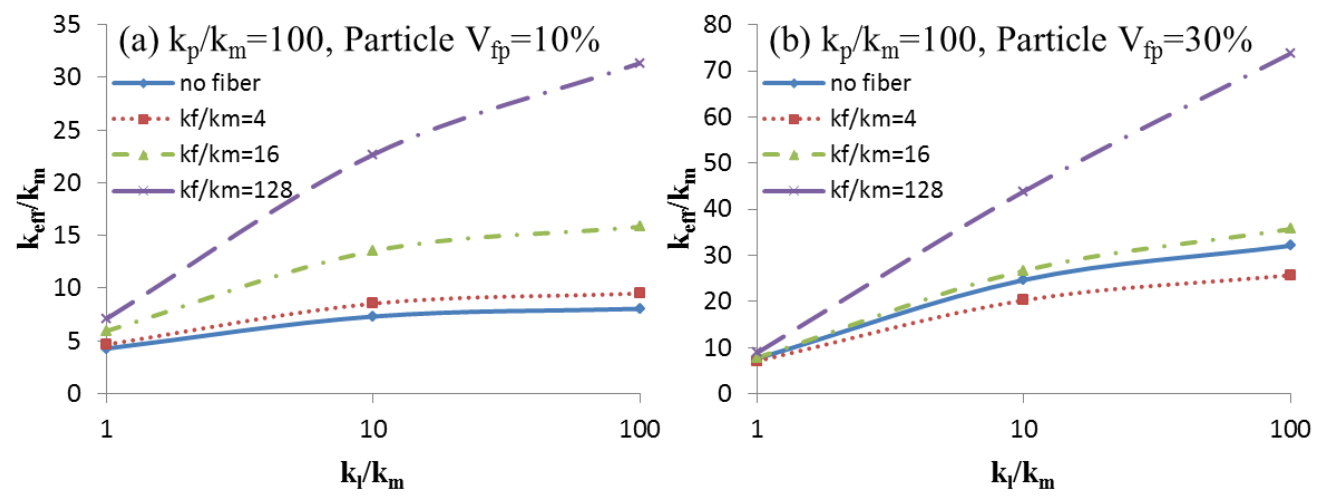

Fig. 12.

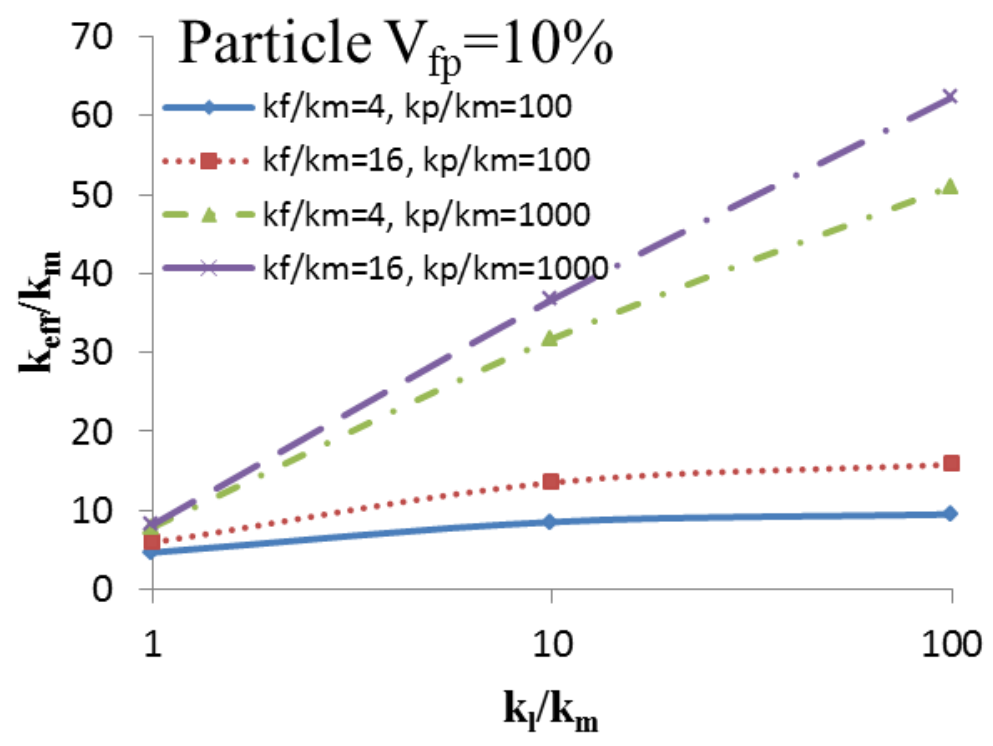

Fig. 13.

Figure captions

Fig. 1. Interlaced yarns in the woven fabric composite with geometric characteristic parameters

Fig. 2. Schematic of the three dimensional woven fabric

Fig. 3. An example of the mesh of a unit cell containing matrix with $10 \%$ volume fraction of randomly distributed particles. Note smallest individual particle volume is the volume of one finite element.

Fig. 4. Finite Element results departure from rule of mixtures with different particle conductivity and volume fractions

Fig. 5. A two-phase woven fabric composite model. (1) Fabric $V_{F}=12.12 \%$; (2) Fabric $V_{F}=38.79 \%$; (3)

Fabric $\mathrm{V}_{\mathrm{F}}=67.87 \%$. Purple and blue fabric represent $\mathrm{X}$ and $\mathrm{Y}$ direction yarns, respectively.

Fig. 6. Refined meshes of the particle embedded woven fabric model which has $10 \%$ particles and $48 \%$ fabric. "0" shows the coarse mesh (155,469 elements), "1" shows the 1-order refined mesh (625,603 elements) where each element is half the size in " 0 " order mesh; in "2" each element is half the size 
of "1" (1,434,214 elements).

Fig. 7. Nondimensional through-thickness thermal conductivity $\boldsymbol{k}_{\boldsymbol{e f f}} / \boldsymbol{k}_{\boldsymbol{m}}$ for the 3D fabric model (fiber volume fraction $\boldsymbol{v}_{\boldsymbol{f}}=\mathbf{3 8 . 7 9} \%$ ) for different $\boldsymbol{k}_{\boldsymbol{f}} / \boldsymbol{k}_{\boldsymbol{m}}$, and $\boldsymbol{k}_{\boldsymbol{p}} / \boldsymbol{k}_{\boldsymbol{m}}$ ratios at 10,20 and 30\% particle volume fractions $v_{f p}$.

Fig. 8. Nondimensional through-thickness thermal conductivity $\boldsymbol{k}_{\boldsymbol{e f f} \mathbf{s}} / \boldsymbol{k}_{\boldsymbol{m}}$ of the 3D fabric model (fiber volume fraction $\boldsymbol{v}_{\boldsymbol{f}}=\mathbf{3 8 . 7 9} \%$ ). for different $\boldsymbol{k}_{\boldsymbol{f}} / \boldsymbol{k}_{\boldsymbol{m}}$, and $\boldsymbol{k}_{\boldsymbol{p}} / \boldsymbol{k}_{\boldsymbol{m}}$ ratios at 10,20 and $30 \%$ particle volume fractions $\boldsymbol{v}_{f p}$.

Fig. 9. Comparison between the through-thickness thermal conductivity predictions $\boldsymbol{k}_{\boldsymbol{e f f} \mathbf{f}} / \boldsymbol{k}_{\boldsymbol{e f f} \mathbf{3}}$ of the 3D fabric model (fiber volume fraction $\boldsymbol{v}_{\boldsymbol{f}}=\mathbf{3 8 . 7 9} \%$ ) with matrix and particle represented as separate phases and particles and matrix represented as a homogenized phase as a function of fiber thermal conductivity for different $\boldsymbol{k}_{\boldsymbol{p}} / \boldsymbol{k}_{\boldsymbol{m}}$ ratios and different particle volume fractions, $\boldsymbol{v}_{\boldsymbol{f} \boldsymbol{p}}$.

Fig. 10. Comparison between the through-thickness thermal conductivity predictions $\boldsymbol{k}_{\boldsymbol{e f f} \mathbf{2}} / \boldsymbol{k}_{\boldsymbol{e f f} \mathbf{3}}$ of the 3D fabric model (particle volume fraction $\boldsymbol{v}_{f p}=\mathbf{1 0} \%$ ) with matrix and particle separate phases and homogenized phase as a function of fiber thermal conductivity for different $\boldsymbol{k}_{\boldsymbol{p}} / \boldsymbol{k}_{\boldsymbol{m}}$ ratios and different fiber volume fractions $\boldsymbol{v}_{f}$

Fig. 11. Conductive skin coated three-phase model with $35 \%$ fiber volume fraction, $10 \%$ particle volume fraction and $10 \%$ skin volume fraction.

Fig. 12. Effective through-thickness thermal conductivity of conductive skin coated three-phase model with $35 \%$ fiber volume fraction and $10 \%$ skin volume fraction. Fiber conductivities $\mathbf{k}_{\mathbf{f}}$ and skin conductivities $\mathbf{k}_{\mathbf{l}}$ are varied with the same thermal conductivity $\mathbf{k}_{\mathbf{p}}$ for particle volume fraction (a) $10 \%$ and (b) $30 \%$.

Fig. 13. The effective thermal conductivity enhancement comparison for different thermal conductivities of particles and fibers with the same skin and particle volume fractions as a function of conductivity of the skin layer 


\section{Reference}

[1] Yu H, Heider D, Advani S. Prediction of effective through-thickness thermal conductivity of woven fabric reinforced composites with embedded particles. Composite Structures. 2015;127:132-40.

[2] Ivanov DS, Ivanov SG, Lomov SV, Verpoest I. Unit cell modelling of textile laminates with arbitrary inter-ply shifts. Composites Science and Technology. 2011;72:14-20.

[3] Hallal A, Younes R, Fardoun F. Review and comparative study of analytical modeling for the elastic properties of textile composites. Composites Part B: Engineering. 2013;50:22-31.

[4] Cho JR, Jee YB, Kim WJ, Han SR, Lee SB. Homogenization of braided fabric composite for reliable large deformation analysis of reinforced rubber hose. Composites Part B: Engineering. 2013;53:112-20.

[5] Gereke T, Döbrich O, Hübner M, Cherif C. Experimental and computational composite textile reinforcement forming: A review. Composites Part A: Applied Science and Manufacturing. 2013;46:1-10.

[6] Bigaud D, Goyhénèche JM, Hamelin P. A global-local non-linear modelling of effective thermal conductivity tensor of textile-reinforced composites. Composites Part A: Applied Science and Manufacturing. 2001;32:1443-53.

[7] Tavana R, Najar SS, Abadi MT, Sedighi M. Meso/macro-scale finite element model for forming process of woven fabric reinforcements. Journal of Composite Materials. 2012;47:2075-85.

[8] Boyina D, Banerjee A, Velmurugan R. Mixed-mode translaminar fracture of plain-weave composites. Composites Part B: Engineering. 2014;60:21-8.

[9] Xu L, Jin CZ, Ha SK. Ultimate strength prediction of braided textile composites using a multi-scale approach. Journal of Composite Materials. 2015;49:477-94.

[10] Gowayed Y, Hwang JC, Chapman D. . Thermal conductivity of textile composites with arbitrary preform structures. Journal of Composites Technology and Research. 1995;17:56-62.

[11] Gori F, Corasaniti S. New model to evaluate the effective thermal conductivity of three-phase soils. International Communications in Heat and Mass Transfer. 2013;47:1-6.

[12] Wang J, Carson JK, North MF, Cleland DJ. A new structural model of effective thermal conductivity for heterogeneous materials with co-continuous phases. International Journal of Heat and Mass Transfer. 2008;51:2389-97.

[13] Wang J, Carson JK, North MF, Cleland DJ. A new approach to modelling the effective thermal conductivity of heterogeneous materials. International Journal of Heat and Mass Transfer. 2006;49:3075-83.

[14] Progelhof RC TJ, Ruetsch RR. Methods for predicting the thermal conductivity of composite systems-a review. Polymer Engineering and Science. 1976;16:615-25.

[15] Das K. Sarit CUSS, Yu Wenhua, Pradeep T. Theoretical Modeling of Thermal Conductivity in Nanofluids. Nanofluids Science and Technology. John Wiley \& Sons, Inc., Hoboken, New Jersey: Wiley; 2007. p. 167-208.

[16] Carvelli V, Poggi C. A homogenization procedure for the numerical analysis of woven fabric composites. Composites Part A: Applied Science and Manufacturing. 2001;32:1425-32.

[17] Zhang C, Xu X. Finite element analysis of 3D braided composites based on three unit-cells models. Composite Structures. 2013;98:130-42. 
[18] Huang L, Wang Y, Miao Y, Swenson D, Ma Y, Yen C-F. Dynamic relaxation approach with periodic boundary conditions in determining the 3-D woven textile micro-geometry. Composite Structures. 2013;106:417-25.

[19] Jacques S, De Baere I, Van Paepegem W. Application of periodic boundary conditions on multiple part finite element meshes for the meso-scale homogenization of textile fabric composites. Composites Science and Technology. 2014;92:41-54.

[20] Lomov SV, Verpoest I, Cichosz J, Hahn C, Ivanov DS, Verleye B. Meso-level textile composites simulations: Open data exchange and scripting. Journal of Composite Materials. 2014;48:621-37.

[21] Wang S, Haldane D, Gallagher P, Liu T, Liang R, Koo JH. Heterogeneously structured conductive carbon fiber composites by using multi-scale silver particles. Composites Part B: Engineering. 2014;61:172-80.

[22] Sheng SZ, Van Hoa S. Three Dimensional Micro-Mechanical Modeling of Woven Fabric Composites. Journal of Composite Materials. 2001;35:1701-29.

[23] Rajkovic V, Bozic D, Popovic M, Jovanovic MT. The Influence of Powder Particle Size on Properties of Cu-A12O3 Composites Science of Sintering. 2009;41:185-92.

[24] Fu S-Y, Feng X-Q, Lauke B, Mai Y-W. Effects of particle size, particle/matrix interface adhesion and particle loading on mechanical properties of particulate-polymer composites. Composites Part B: Engineering. 2008;39:933-61.

[25] Porfiri M, Nguyen NQ, Gupta N. Thermal conductivity of multiphase particulate composite materials. Journal of Materials Science. 2008;44:1540-50.

[26] Ferguen N, Cogne C, Bellenger E, Guessasma M, Pelegris C. A numerical model for predicting effective thermal conductivities of alumina/Al composites. Journal of Composite Materials. 2012;47:3311-21.

[27] Leung SN, Khan MO, Chan E, Naguib H, Dawson F, Adinkrah V, et al. Analytical modeling and characterization of heat transfer in thermally conductive polymer composites filled with spherical particulates. Composites Part B: Engineering. 2013;45:43-9.

[28] Nguyen Dinh Duc LVB, Nguyen Tien Dac. Determining thermal expansion coefficients of three-phase fiber composite material reinforced by spherical particles. VNU Journal of Science, Mathematics - Physics. 2008;24:57-65.

[29] Buonanno G, Carotenuto A. The effective thermal conductivity of a porous medium with interconnected particles. International Journal of Heat and Mass Transfer. 1997;40:393-405.

[30] Moosavi A SP. The effective conductivity of three-phase composite materials with circular cylindrical inclusions. Journal of Physics D: Applied Physics. 2003;36:1644-50. 